\title{
Adaptation of MANET topology to monitor dynamic phenomena clouds
}

\author{
Mateusz Krzysztoń $^{1,2}$ and Ewa Niewiadomska-Szynkiewicz ${ }^{1,2}$ \\ ${ }^{1}$ Research and Academic Computer Network (NASK) \\ ul. Kolska 12, 01-045 Warsaw, Poland \\ Email: mateusz.krzyszton@nask.pl \\ ${ }^{2}$ Institute of Control and Computation Engineering \\ Warsaw University of Technology \\ ul. Nowowiejska 15/19, 00-665, Warsaw, Poland \\ Email: ens@ia.pw.edu.pl
}

\begin{abstract}
The paper is concerned with the application of mobile ad hoc networks to phenomena clouds boundary detection and tracking. Self-organizing, coherent networks comprised of sensors and radio transceivers that maintain a continuous communication with each other and a central operator are considered. The attention is focused on the methodology for determining the temporarily optimal network topology for detecting the boundary of a cloud that can change its shape in time. We introduce several measures for assessment a quality of a network topology and propose a computing scheme for detection topology that is the optimal one at a given time. The utility and efficiency of the proposed methodology was justified through simulation experiments.
\end{abstract}

\section{INTRODUCTION}

$\mathbf{P}$ HENOMENA clouds are objects covering significant area and characterized by nondeterministic, dynamic variations of shape, size, speed, and direction of motion along multiple axes [1]. The examples of phenomena cloud can be not only environmental disasters as oil spill, toxic heavy gas cloud, flood or forest fire, but also moving group of people. In general, in case of one of the aforementioned disasters the extensive monitoring of the area of interest is necessary to manage the evacuation of people from a hazardous zone, track the propagation of a given cloud and finally, neutralize the threat.

Nowadays, mobile ad hoc networks (MANETs) are becoming increasingly popular solutions for environmental monitoring. MANET is comprised of mobile devices, which are usually equipped with GPS receivers, various detectors and radio transceivers that enable wireless communication within the network. The devices can autonomously and dynamically self-organize by changing their positions and roles into temporal networks. In general, in emergency situation MANET should not rely on external communication system as it can be damaged or congested due to the disaster. Hence, the network needs to maintain connectivity among the working set of devices and a base station. Numerous approaches to the connectivity maintenance have been proposed in the literature [2]-[6].
MANETs are widely used to cover a region of interest (ROI) [7], [8]. Phenomena cloud is a special type of ROI due to its dynamic character. Results of research on adaptation of a sensing network topology to variant phenomena cloud boundary was comprehensively described in [9], [10].

In this paper we focus on measuring quality of a network topology taking into account multiple spatial criteria. Spatial topology analysis was widely used in MANET. In [11] dense and sparse regions are identified to limit rebroadcasting packets in flooding routing protocol. Routing protocols depending on nodes' location are described in [12]. Moreover, topology analysis can support a clustering of network. Topology Adaptive Spatial Clustering that divides the network into a locally isotropic, non-overlapping clusters by creating a set of weights that encode distance, connectivity and density information within the neighborhood of each node is described in [13]. In [14] prediction of the existence of a link given the present distance between a pair of nodes and their relative speed is proposed. The prediction is based on two topology metrics: an expected link lifetime and an expected link change rate.

In this paper we define several measures (spatial parameters) that can be used to assess the quality of a current network topology. Moreover, we propose a methodology that can be used to detect the acceptable topology — the best possible configuration of a network for monitoring a given cloud at a given time step. The technique for phenomena cloud boundary detection described in [6] is extended with the analysis of a topology dynamics. The presented approach allows to increase the efficiency of detection of clouds with unknown and irregular shapes.

The article is organized as follows. First, computing scheme for detection of boundary of area covered by a phenomena cloud with mobile sensors is described. Then we introduce several measures for assessing quality of a given sensing topology and statistical tools for analyzing the variability of these measures. Next, we introduce computing scheme for detecting the temporrily optimal sensing topology. Finally, the results of applications of our method to detect the heavy 
gas cloud are presented and discussed. Two more and less advanced gas dispersion models were taken into account: the box model [15] and the advance model provided in SLAB [16].

\section{PROBLEM FORMULATION}

Let us consider the network $\mathcal{G}=(\mathcal{V}, \mathcal{E})$ comprised of a set $\mathcal{V}$ of $N$ mobile nodes (unmanned vehicles or mobile robots) $D_{i}, i=1, \ldots, N$ operating in the workspace. $\mathbf{o}_{i}=\left[x^{i}, y^{i}\right]$ denotes the reference point of $D_{i}$ (e.g. an antenna location). It is assumed, that each node $D_{i}$ is equipped with a punctual detector for sensing a given phenomena, radio transceiver (with radio range $r_{t}$ ) and a positioning system, e.g. GPS receiver. Let us define our sensing network

$$
\begin{gathered}
\mathcal{V}=\left\{D_{i}, i=1, \ldots, N\right\}, \\
\mathcal{E}=\left\{\left(D_{i}, D_{j}\right), d_{j}^{i} \leq r_{t}, i, j=1, \ldots, N, i \neq j\right\},
\end{gathered}
$$

where $\left(D_{i}, D_{j}\right)$ is a bidirectional link between a pair of nodes $D_{i}$ and $D_{j}$ and $d_{j}^{i}$ is the Euclidean distance between the reference points $\mathbf{o}_{i}$ and $\mathbf{o}_{j}$ of nodes $D_{i}$ and $D_{j}$. We assume that each node can freely change both its position and role according to its knowledge about an environment and a network. It can move with the speed $v \in\left[v_{\min }, v_{\max }\right]$ in desirable direction.

Let us divide the network $\mathcal{G}$ into $K$ separated clusters $\mathcal{V}_{k}$, $k=1, \ldots, K$ of devices:

$$
\begin{aligned}
& \mathcal{V}_{1} \cup \mathcal{V}_{2} \cup \ldots \cup \mathcal{V}_{K}=\mathcal{V}, \\
& \mathcal{V}_{1} \cap \mathcal{V}_{2} \cap \ldots \cap \mathcal{V}_{K}=\emptyset .
\end{aligned}
$$

We assign $D_{H_{k}} \in \mathcal{V}_{k}$ a role of the $k$ th cluster head, $k=$ $1, \ldots, K$, and select one of cluster heads $D_{H}$ to be a head of the whole network, $D_{H} \in D_{H_{1}}, \ldots, D_{H_{K}}$.

It is assumed that the network can self-organize to accomplish a given task. The task considered in this paper is to sense boundaries of a given phenomena cloud to estimate a size and a shape of this cloud. The scheme for robot-assisted sensors deployment was developed and described in [6]. In this work we focus on the last phase of the deployment, i.e. boundary detection. We assume that at least one device detected a cloud and the cloud center $\Psi$ was estimated by $D_{H}$ based on known locations of those network nodes $\mathcal{V}^{\prime}$, which sensors detected the phenomena (i.e. nodes located inside the cloud):

$$
\Psi=\frac{\sum_{D_{i} \in \mathcal{V}^{\prime} \mathbf{o}_{i}}}{\left|\mathcal{V}^{\prime}\right|}
$$

To determine the boundary of a given cloud with high accuracy we need measurements from sensors that should be evenly deployed on the boundary (Fig. 1). Hence, our goal is to create a sensing network with evenly distributed nodes. Moreover, the permanent communication within the network has to be maintained to exchange information about topology and current measurements between nodes and report measurements to a base station.

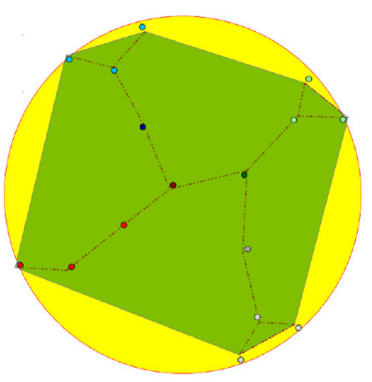

(a)

Fig. 1: Even deployment of network clusters on the cloud boundary;

The nodes mobility model incorporates the concept of an artificial potential. The artificial potential function is constructed as a sum of repulsive and attractive potentials [17]. Every assumed time step each $D_{i} \in \mathcal{V}_{m}$ solves the following optimization problem to calculate its new position:

$$
\begin{array}{r}
\min _{\mathbf{c}^{i}}\left[U^{i}=U_{c}^{i}+\sum_{D_{j} \in S_{i}, D_{j} \in \mathcal{V}_{m}} U_{j}^{i}+\sum_{k \in I C_{m}} U_{k}^{i}\right. \\
=\alpha_{c}\left(\frac{\bar{d}_{c}^{i}}{d_{c}^{i}}-1\right)^{2}+\sum_{D_{j} \in S_{i}, D_{j} \in \mathcal{V}_{m}} \beta_{j}\left(\frac{\bar{d}_{j}^{i}}{d_{j}^{i}}-1\right)^{2} \\
\left.+\sum_{k \in I C_{m}} \gamma_{k}\left(\frac{\bar{d}_{k}^{i}}{d_{k}^{i}}-1\right)^{2}\right] .
\end{array}
$$

In the above formulation artificial potential function $U^{i}$ consists of the potential $U_{c}^{i}$ between $D_{i}$ and the cloud centroid $\Psi$, a sum of potentials $U_{j}^{i}$ between $D_{i}$ and its neighboring nodes $D_{j}, D_{j} \in S_{i}=\left\{D_{j}:\left(D_{i}, D_{j}\right) \in \mathcal{E}, D_{j} \in \mathcal{V}_{m}\right\}$ and a sum of potentials $U_{k}^{i}$ between $D_{i}$ and neighboring clusters $I C_{m} . \alpha_{c} \geq 0, \beta_{j} \geq 0, \gamma_{k} \geq 0$ denote weighting factors, $d_{c}^{i}$ is an Euclidean distance between $\mathbf{o}_{i}$ and estimated centroid $\Psi$ of the cloud, $\bar{d}_{c}^{i}=\max _{D_{i} \in \mathcal{V}^{\prime}} d_{c}^{i}+w_{1}$, where $w_{1}>0$ denotes a distance margin (arbitrary selected), $\bar{d}_{j}^{i} \leq r_{t}$ is a reference distance between two neighboring nodes $D_{i}$ and $D_{j}$. In the last part of eq. (6) $I C_{m}$ is a set of indexes of two closest neighboring clusters of the $m$ th cluster that contains $D_{i}$, defined as follows:

$$
\begin{aligned}
& I C_{m}=\left\{\underset{\mathcal{V}_{j} \neq \mathcal{V}_{m}}{\arg \min } \varangle\left(\mathcal{V}_{m}, \mathcal{V}_{j}\right)\right\} \cup\left\{\underset{\mathcal{V}_{j} \neq \mathcal{V}_{m}}{\arg \max } \varangle\left(\mathcal{V}_{m}, \mathcal{V}_{j}\right)\right\} \\
& \varangle\left(\mathcal{V}_{m}, \mathcal{V}_{j}\right)= \\
& \begin{cases}\arccos \frac{\overrightarrow{\Psi \mathbf{c}_{m}} \cdot \overrightarrow{\Psi \mathbf{c}_{j}}}{\left|\Psi \mathbf{c}_{m}\right| \cdot\left|\Psi \mathbf{c}_{j}\right|} & {\left[\overrightarrow{\Psi \mathbf{c}_{m}} \times \overrightarrow{\Psi \mathbf{c}_{j}}\right]_{z} \geq 0} \\
2 \Pi-\arccos \frac{\overrightarrow{\Psi \mathbf{c}_{m}} \cdot \overrightarrow{\Psi \mathbf{c}_{j}}}{\left|\Psi \mathbf{c}_{m}\right| \cdot\left|\Psi \mathbf{c}_{j}\right|} & {\left[\overrightarrow{\Psi \mathbf{c}_{m}} \times \overrightarrow{\Psi \mathbf{c}_{j}}\right]_{z}<0}\end{cases} \\
& \mathbf{c}_{m}=\frac{\sum_{D_{i} \in \mathcal{V}_{m}} \mathbf{o}_{i}}{\left|\mathcal{V}_{m}\right|}
\end{aligned}
$$


where $[\vec{q}]_{z}$ is z-component of vector $\vec{q} \cdot d_{k}^{i}$ is a distance between $\mathbf{o}_{i}$ and the centroid of the $k$ th cluster $c_{k}$ and $\bar{d}_{k}^{i}$ is an average distance between two neighboring clusters of the $m$ th cluster (clusters with indexes from the set $I C_{m}$ ) increased by a small margin $w_{2}$ slightly greater than 0 :

$$
\bar{d}_{k}^{i}=\frac{\sum_{k \in I C_{m}} d_{k}^{i}}{2}+w_{2}, \quad w_{2}>0 .
$$

Detailed description of the mentioned above network deployment scheme can be found in [6]. The result of the boundary detection phase is even distribution of nodes on the boundary of an area covered by a given phenomena cloud (Fig. 1). Due to the dynamic changes of the phenomena clouds the next phase is boundary tracking - nodes move and follow the boundary, keeping internode and intercluser distances. The aim of the research described in this paper was to develop a methodology for detecting the temporarily optimal topology for boundary tracking and switch to the boundary tracking phase. The definitions of measures that we used to evaluate the quality of a given topology are provided in the next section.

\section{NETWORK TOPOLOGY QUALITY MEASURES}

Let us consider a network defined in (1)-(4). To create a topology that allows to determine a cloud boundary at a given time and maintain the permanent connectivity we have to perform the following operation (see eq. 6):

- move all devices towards the cloud boundary (increase distance between clusters and the center of cloud);

- expand an area monitored by clusters (increase distances between nodes within cluster);

- deploy clusters on the cloud boundary (as evenly as possible).

Various measures can be used to evaluate the quality of a given MANET at a given time. Taking into account the above operations the following ones can be defined:

- distance between a centroid of the $m$ th cluster to the estimated centroid of a cloud

$$
d_{c}^{m}=\left\|c_{m}-\Psi\right\|_{2} .
$$

The distance is increased as long as the $m$ th cluster nodes do not reach the cloud boundary. The bigger distance is the better topology is.

- $m$ th cluster diameter

$$
\phi_{m}=\max _{D_{i}, D_{j} \in \mathcal{V}_{m}} d_{j}^{i} .
$$

The bigger $\phi_{m}$ is the bigger area is monitored by the $m$ th cluster.

- standard deviation of angles between neighboring clusters

$$
\sigma_{\varangle}=\sqrt{\frac{\sum_{m=1}^{K}\left(\varangle_{m}-\mu_{\varangle}\right)^{2}}{K-1}}, \mu_{\varangle}=\frac{\sum_{m=1}^{K} \varangle_{m}}{K},
$$

where $\varangle_{m}=\varangle\left(\mathcal{V}_{m}, \mathcal{V}_{j}\right)$ (Fig. 2) is an angle between the cluster $\mathcal{V}_{m}$ and its the closest neighboring cluster $\mathcal{V}_{j}$ :

$$
j=\underset{k \neq m}{\arg \min } \varangle\left(\mathcal{V}_{m}, \mathcal{V}_{k}\right) .
$$

For evenly distributed clusters $\sigma_{\varangle} \approx 0$.

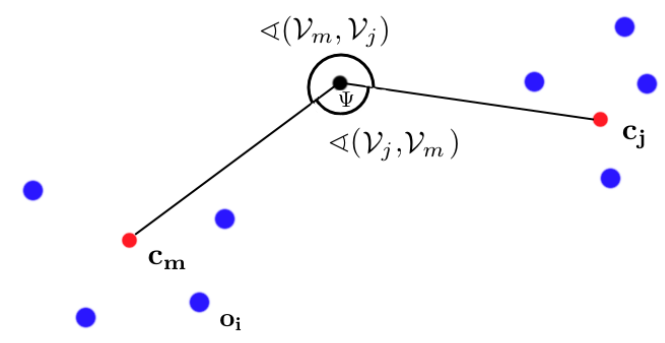

Fig. 2: The angle $\varangle\left(\mathcal{V}_{m}, \mathcal{V}_{j}\right)$ between a cluster $V_{m}$ and a cluster $V_{j}$ and the angle $\varangle\left(\mathcal{V}_{j}, \mathcal{V}_{m}\right)$ between a cluster $V_{j}$ and a cluster $V_{m}$.

\section{Measures VARIABILITy ANALysis}

Changes of measures defined in (11)-(13) involve changes in the topology of a given sensing network. However, to monitor and analyze network topology dynamics we need values of the defined measures from a time interval. Let us assume that $\mu\left(t_{i}\right)$ is a value of the measure $\mu$ calculated at $t_{i}\left(\mu \in\left\{\sigma_{\varangle}, \phi_{m}, d_{c}^{m} \mid m=1, \ldots, K\right\}\right)$ and $X^{\mu}(t)=<\mu(t-M+1), \ldots, \mu\left(t_{i}\right), \ldots, \mu(t)>$ is a vector of values of the measure $\mu$ calculated at last $M$ time steps. The goal of our analysis is to detect does a given measure

- increase (growth);

- decrease (drop);

- change slightly (stabilization);

- change very dynamically and chaotically (instability).

In brackets names of the variability types were introduced. It should be emphasized that detection of variability type has to be done a priori (in real time), with no information about future measurement values. Moreover, the window size $M$ that is arbritraly determined can influence the results of an analysis.

To analyze variability of the values of the vector $X^{\mu}(t)$ following simple statistical measures were proposed:

- peak-to-peak amplitude $A$ :

$$
A=\max X^{\mu}(t)-\min X^{\mu}(t)
$$

- Pearson's correlation $\rho_{X, t}$ :

$$
\begin{gathered}
\rho_{X, t}= \\
\frac{\sum_{i=1}^{M}(\mu(t-M+i)-\bar{\mu})\left(i-\frac{M+1}{2}\right)}{\sqrt{\sum_{i=1}^{M}(\mu(t-M+i)-\bar{\mu})^{2}} \sqrt{\sum_{i=1}^{M}\left(i-\frac{M+1}{2}\right)}}, \\
\bar{\mu}=\frac{\sum_{i=1}^{M} \mu(t-M+i)}{M}
\end{gathered}
$$

- trend direction coefficient $t r_{a}$ (trend line given as $f(x)=$ $\left.\operatorname{tr}_{a} x+b\right)$ :

$t r_{a}=$

$$
\frac{M \sum_{i=1}^{M} \mu(t-M+i) i-\sum_{i=1}^{M} i \sum_{i=1}^{M} \mu(t-M+i)}{M \sum_{i=1}^{M} i^{2}-\left(\sum_{i=1}^{M} i\right)^{2}} .
$$


Based on these measures we can determine whether in time $t$ the vector $X^{\mu}(t)$ induces one of the previously defined variability types:

- growth and drop:

- Pearson's correlation; if $\rho_{X, t} \in\left(\rho_{H}, 1\right]$ a measure $X^{\mu}(t)$ is continually increasing with time; if $\rho_{X, t} \in\left[-1, \rho_{L}\right)$ the value is continually decreasing, where:

$$
\rho_{H} \geq 0.5 ; \rho_{L} \leq-0.5
$$

- trend direction coefficient; if $t r_{a} \geq t r_{H}$ the measure is increasing significantly, else if $t r_{a} \leq t r_{L}$ the measure $X^{\mu}(t)$ is decreasing significantly, where:

$$
t r_{H}>0 ; t r_{L}<0 \text {. }
$$

\section{- stabilization:}

- peak-to-peak amplitude; if $A<A_{L}$ value of measure $X^{\mu}(t)$ does not change significantly;

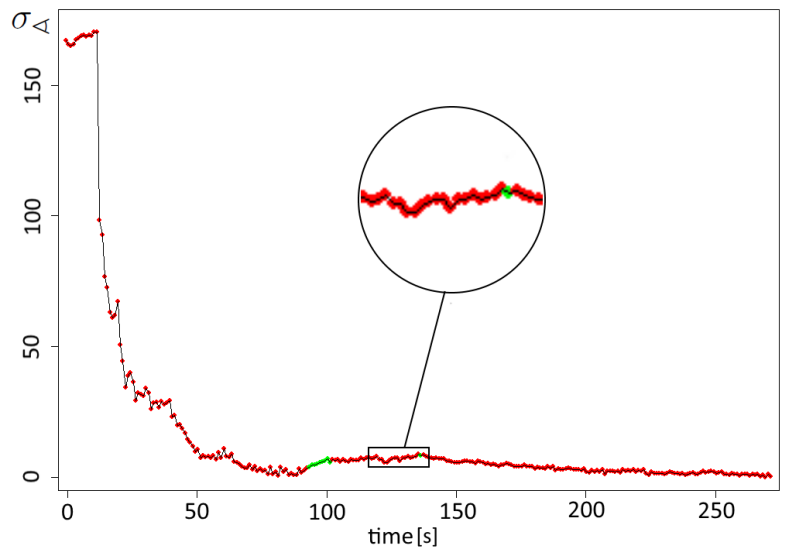

(a)

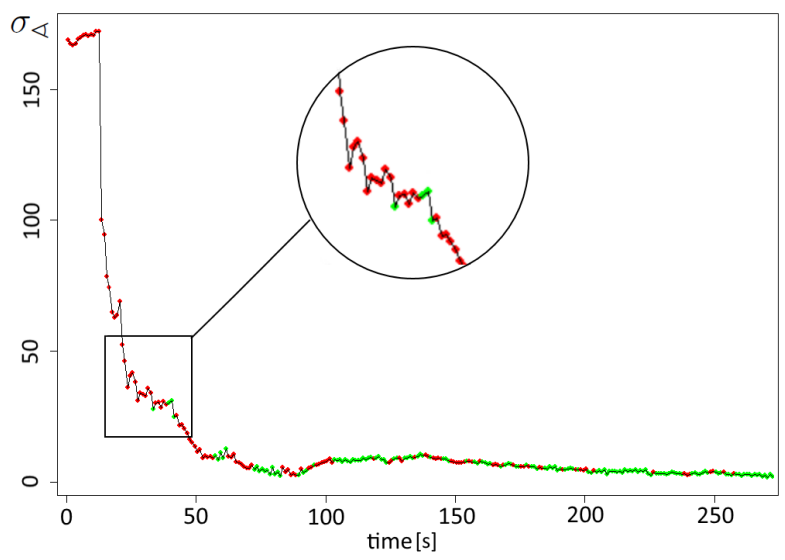

(c)
- Pearson's correlation; if $\rho_{X, t} \in\left(-\rho_{M}, \rho_{M}\right)$ the measure $X^{\mu}(t)$ is neither continually decreasing nor continually increasing, where:

$$
\rho_{M} \in(0,0.7]
$$

- instability:

- peak-to-peak amplitude; if $A>A_{H}$ value of property does change significantly $\left(A_{H}>>A_{L}\right)$

- Pearson's correlation - as in stabilization.

The simulation study was performed to determine the values of threshold values $\rho_{H}, \rho_{L}, \rho_{M}, t r_{L}, t r_{H}, A_{L}, A_{H}$. Within the study an experiment described in [6] (for $K=4$ ) was performed for various values of $v_{\max }, v_{\max } \in\left\{1 \frac{\mathrm{m}}{\mathrm{s}}, 5 \frac{\mathrm{m}}{\mathrm{s}}, 20 \frac{\mathrm{m}}{\mathrm{s}}\right\}$. The estimated values of threshold values are presented in Table I. It was observed that values of some thresholds $\left(t r_{H}, \operatorname{tr}_{L}, A_{L}, A_{H}\right)$ depend on the maximal velocity of nodes $v_{\max }$ as the higher velocity involves the bigger changes of measures every time step.

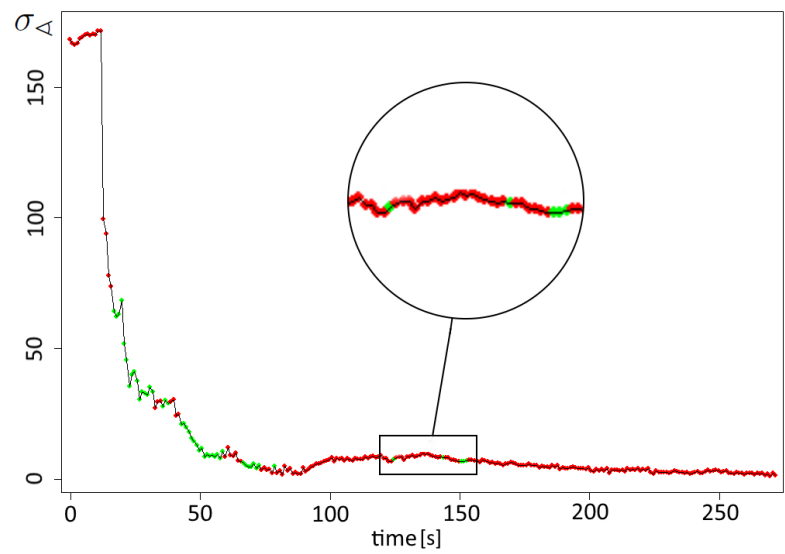

(b)

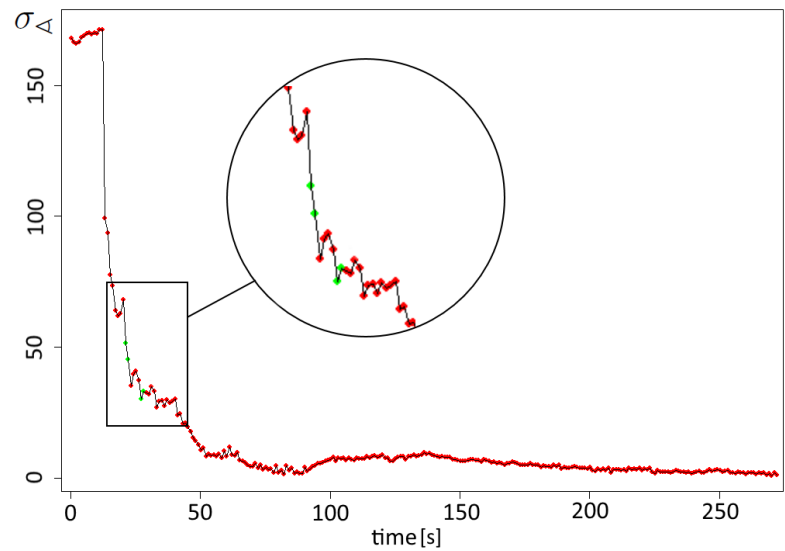

(d)

Fig. 3: Detection (green color) of (a) growth, (b) drop, (c) stabilization and (d) instability variability type of $\sigma_{\varangle}$ property during the network deployment. The less visible parts of graphs in which detection occurred were enlarged. 
TABLE I: Values of threshold parameters

\begin{tabular}{|c|c|c|c|c|c|c|c|}
\hline \multicolumn{8}{|c|}{$\sigma_{\varangle}$ measure } \\
\hline \multicolumn{2}{|c|}{ stabilization } & \multicolumn{2}{|r|}{ drop } & \multicolumn{2}{|r|}{ growth } & \multicolumn{2}{|r|}{ instability } \\
\hline $\bar{M}$ & 5 & $M$ & 8 & $M$ & 8 & $M$ & 5 \\
\hline$\rho_{M}$ & 0.7 & $\rho_{L}$ & -0.6 & $\rho_{H}$ & 0.6 & $\rho_{M}$ & 0.6 \\
\hline$\overline{A_{L}}$ & $1+0.1 v_{\max }$ & $\overline{t r_{L}}$ & $-0.05-0.01 v_{\max }$ & $\overline{t r} r_{H}$ & $0.05+0.01 v_{\max }$ & $\overline{A_{H}}$ & $3+0.3 v_{\max }$ \\
\hline \multicolumn{8}{|c|}{$d_{c}^{m}$ measure } \\
\hline \multicolumn{2}{|c|}{ stabilization } & \multicolumn{2}{|r|}{ drop } & \multicolumn{2}{|r|}{ growth } & \multicolumn{2}{|r|}{ instability } \\
\hline$M$ & 5 & $\bar{M}$ & 6 & $\bar{M}$ & 8 & $M$ & 6 \\
\hline$\rho_{M}$ & 0.7 & $\rho_{L}$ & -0.7 & $\rho_{H}$ & 0.7 & $\rho_{M}$ & 0.5 \\
\hline$A_{L}$ & $0.5 v_{\max }$ & $t r_{L}$ & -0.1 & $t r_{H}$ & $0.1+0.1 v_{\max }$ & $A_{H}$ & $1+0.8 v_{\max }$ \\
\hline \multicolumn{8}{|c|}{$\phi_{m}$ measure } \\
\hline \multicolumn{2}{|c|}{ stabilization } & \multicolumn{2}{|r|}{ drop } & \multicolumn{2}{|r|}{ growth } & \multicolumn{2}{|r|}{ instability } \\
\hline$M$ & 4 & $M$ & 4 & $M$ & 4 & $M$ & 4 \\
\hline$\rho_{M}$ & 0.5 & $\rho_{L}$ & -0.7 & $\rho_{H}$ & 0.7 & $\rho_{M}$ & 0.5 \\
\hline$A_{L}$ & $1.6 v_{\max }$ & $\overline{t r_{L}}$ & $-0.4-0.1 v_{\max }$ & $\operatorname{tr}_{H}$ & $0.4+0.1 v_{\max }$ & $\overline{A_{H}}$ & $3.2 v_{\max }$ \\
\hline
\end{tabular}

The results of application of the proposed variability type detection scheme for the measure $\sigma_{\varangle}$ and the threshold values calculated for $v_{\max }=20 \frac{\mathrm{m}}{\mathrm{s}}$ (see Table I) are depicted in Figures 3a-3d. Each figure corresponds to one variability type: growth, drop, stabilization or instability. The time steps in which a given variability type was detected are marked with a green color. It can be observed that in most cases the variability types were detected correctly. However, in some cases (see Fig. $3 \mathrm{~b}, t=125$ ) a variability type was detected too late. It was caused by too long observation time window.

\section{TEMPORARILY OPTIMAL TOPOLOGY DETECTION}

Summing up, the aforementioned considerations. The aim is to create the network topology that seems to be optimal to measure the boundary of a cloud at a given time. Exactly, due to the dynamic nature of the monitored cloud our goal reduces to the detection of the time step $t$ at which we claim that the network configuration is stable or the local optimum for $t^{*}, t^{*} \in[t-M+1, t-1]$ was reached and we assume that all changes in the nearest future involve its deterioration. Such network topology in time $t$ is called temporarily optimal.

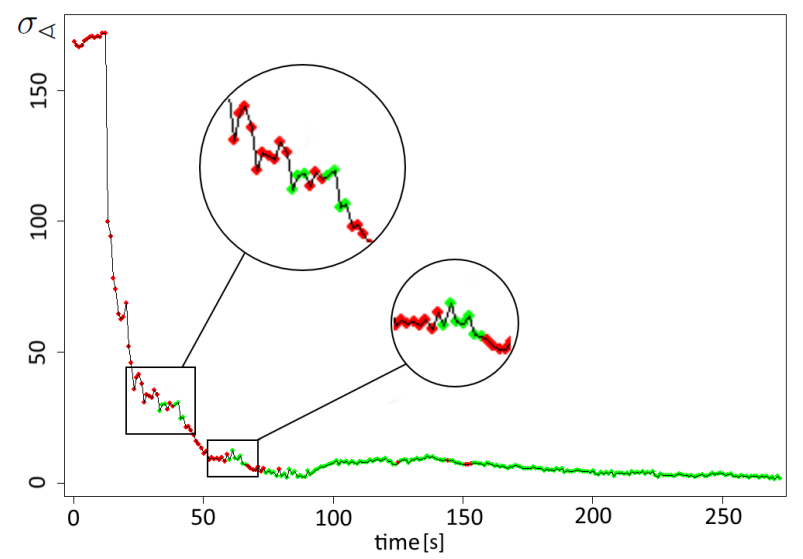

Fig. 4: Time steps in which the variability type of measure $\sigma_{\varangle}$ is stabilization or growth.
The decision whether the topology is temporarily optimal is made based on the observed variability types of all defined measures:

a) the topology is stable for:

- $\sigma_{\varangle}$ - type stabilization;

- $d_{c}^{m}, m \in\{1, \ldots, K\}$ - type stabilization;

- $\phi_{m}, m \in\{1, \ldots, K\}$ - type stabilization;

b) the topology will be worse in the nearest future for:

- $\sigma_{\varangle}$ - type stabilization or growth;

- $d_{c}^{m}, m \in\{1, \ldots, K\}$ - type stabilization or drop;

- $\phi_{m}, m \in\{1, \ldots, K\}$ - type stabilization or drop;

but not case a).

The head of $m$ th cluster $D_{H_{m}}$ detects the variability type of the measures $d_{c}^{m}$ and $\phi_{m}$, whereas the head of the whole network $D_{H}$ detects the variability type of the measure $\sigma_{\varangle}$. The calculations are performed repetitively with the repetition time equal to $\Delta t$. If none of the variability types is detected for measure $\mu$ in time $t$ based on $X^{\mu}(t)$ the variability type of this measure in time $t$ is the same as in time $t-\Delta t$ (we assume that in the beginning the variability type of each measure is instability). Each of cluster heads $D_{H_{m}}$ sends information about variability types of $d_{c}^{m}$ and $\phi_{m}$ to the network head. Exemplary detection of time steps in which variability type of measure $\sigma_{\varangle}$ for the aforementioned example (Fig. 3) is stabilization or growth is depicted in Fig. 4.

Due to the large number of requirements regarding the number of measures $(2 * K+1)$ that have to be taken into account during the decision problem and dynamics of a phenomena fulfilling all of the requirements is too rigorous. Therefore, we propose two distributed strategies.

- Strategy 1: The topology is temporarily optimal if:

- variability type of $\sigma_{\varangle}$ is stabilization or growth;

- exists at least $r_{1}$ clusters for which variability type of both $d_{c}^{m}$ and $\phi_{m}$ is stabilization or drop.

- Strategy 2: The topology is temporarily optimal if:

- variability type of $\sigma_{\varangle}$ is stabilization or growth;

- exists at least $r_{2}$ clusters for which variability type of $d_{c}^{m}$ is stabilization or drop; 


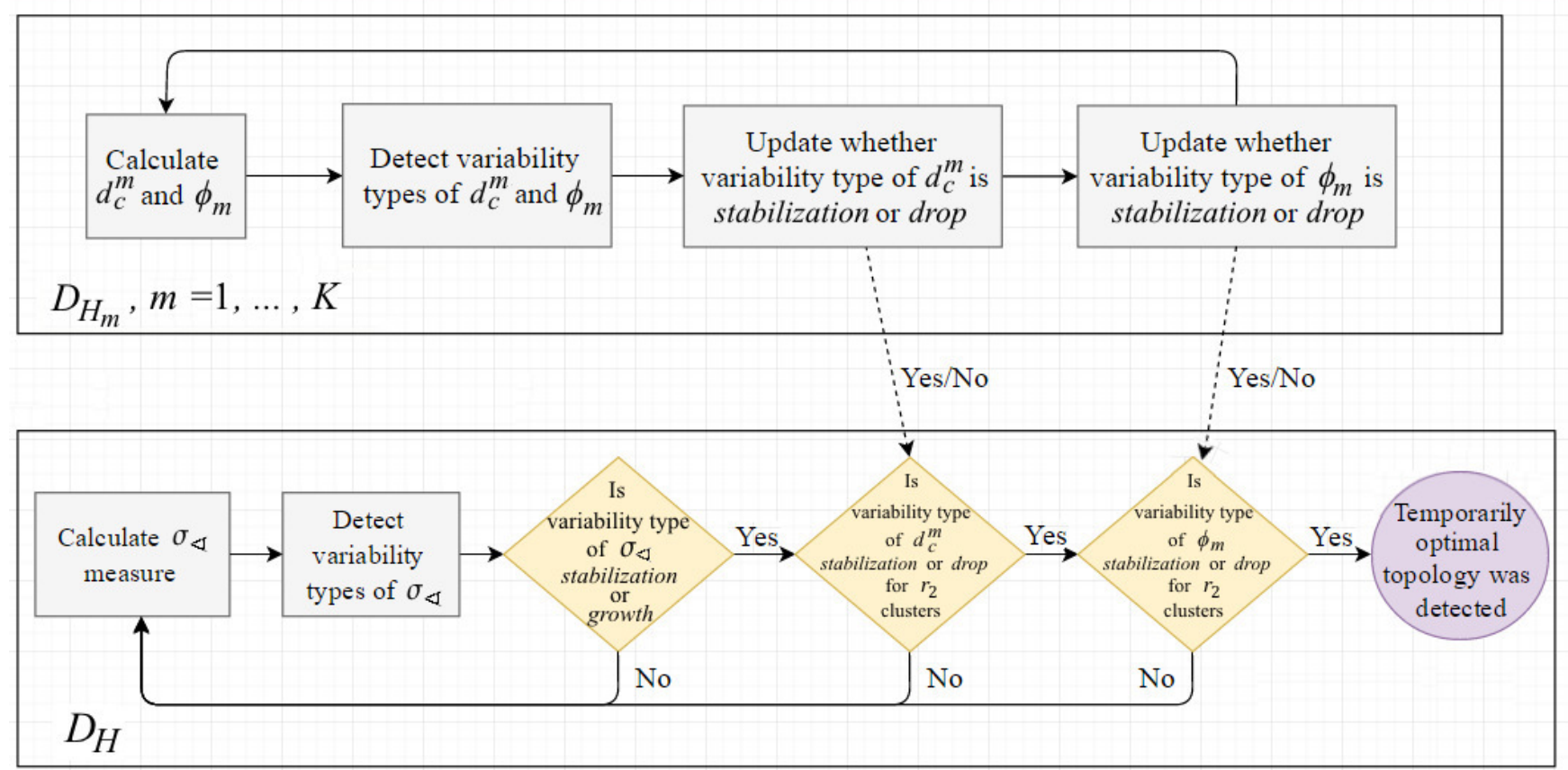

Fig. 5: Temporarily optimal topology detection - Strategy 2. With the dotted arrows communication between the cluster head $D_{H_{m}}$ and the network head $D_{H}$ is shown.

- exists at least $r_{2}$ clusters for which variability type of $\phi_{m}$ is stabilization or drop.

The scheme of making decision according to Strategy 2 . is presented in Fig. 5. For $r_{1}=r_{2}$ the Strategy 1 is more rigorous. The results of experiments for various values of $r_{1}$ and $r_{2}$ are presented and discussed in the next section.

\section{EXPERIMENTAL VERIFICATION}

The performance of our methodology for cloud boundary detection was verified through simulation experiment. The task was to create a sensing MANET for monitoring uncontrolled instantaneous release of vapor LNG. Due to a low temperature of the release the created cloud was heavier-than-air. Thus, it moved close to the ground. The parameters of the released material and ambient environment are presented in Table II. The dispersion of cloud was simulated using the SLAB simulator [16]. The sensing network was built by 16 devices divided into 4 clusters. The maximal velocity $v_{\max }$ of each node was equal to $10 \frac{\mathrm{m}}{\mathrm{s}}$.

The method for detection of acceptable topology was tested for both proposed strategies and for different values of $r_{1}, r_{2} \in\{2,3,4\}$. For both strategies for $r_{1}=r_{2}=3$ the optimal topology was detected at the same time $t=102$. However, for $r_{1}=r_{2}=2$ the results obtained for both strategies were different: $t=100$ for Strategy 1 and $t=52$ for Strategy 2. In the extreme case $r_{1}=r_{2}=K=4$ (all requirements have to be fulfilled) the consensus was not reached until $t=220$.

Fig. 6 shows the process of forming the network topology for cloud boundary monitoring. The results of experiment indicate that at $t=52$ (Fig. 6a) the topology meets basic
TABLE II: The parameters of the released material and ambient environment in the verification scenario

\begin{tabular}{|c|c|c|}
\hline Name & Value & Units \\
\hline Molecular weight & 0.01604 & $\mathrm{~kg}$ \\
\hline Vapor heat capacity at constant pressure & 2238 & $\frac{\mathrm{J}}{\mathrm{kg} * \mathrm{~K}}$ \\
\hline Boilling point temperature & 111.7 & $\mathrm{~K}$ \\
\hline Heat of vaporization & 509900 & $\frac{\mathrm{J}}{\mathrm{kg}}$ \\
\hline Liquid heat capacity & 3348.5 & $\frac{\mathrm{J}}{\mathrm{kg}}$ \\
\hline Liquid density of source material & 424.1 & $\frac{\mathrm{kg}}{\mathrm{m}^{3}}$ \\
\hline Temperature of source material & 111.7 & $\mathrm{~K}$ \\
\hline Source area & 900 & $\mathrm{~m}$ \\
\hline Instantaneous source mass & 6000 & $\mathrm{~kg}$ \\
\hline Surface roughness height & 0.01 & $\mathrm{~m}$ \\
\hline Ambient measurement height & 2.88 & $\mathrm{~m}$ \\
\hline Ambient wind speed & 1.92 & $\frac{\mathrm{m}}{\mathrm{s}}$ \\
\hline Ambient temperature & 306 & $\mathrm{~K}$ \\
\hline Relative humidity & 4.6 & $\%$ \\
\hline
\end{tabular}

requirements - at least one node of each cluster is on the boundary, distance between clusters (except green and gray) are significant and nodes within clusters are rather dispersed. However, at time $t=100$ clusters are much more evenly dispersed on the boundary (Fig. 6b). Finally, at $t=220$ (Fig. 6d) the topology is slightly better (better dispersion of nodes within clusters). Furthermore there is no improvement comparing to topology created at $t=180$ (Fig. 6c).

According to the results of the experiment it can be induced that $r_{2}=\frac{K}{2}$ for Strategy 2 is too weak requirement and fulfilling all requirements $\left(r_{1}=r_{2}=K\right)$ may delay the detection of temporarily optimal topology unnecessarily. Thus, 


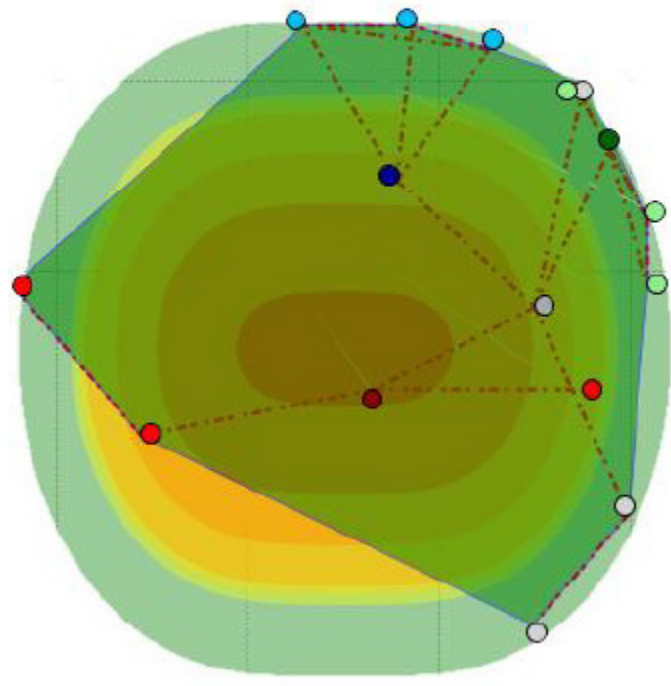

(a)

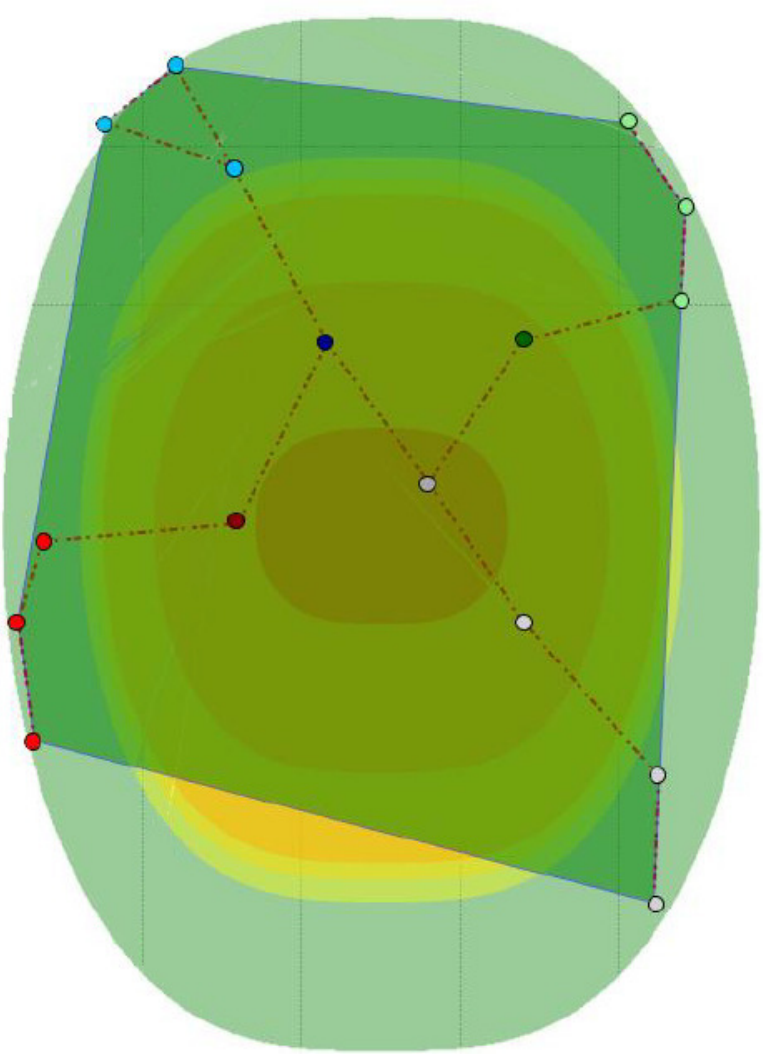

(c)

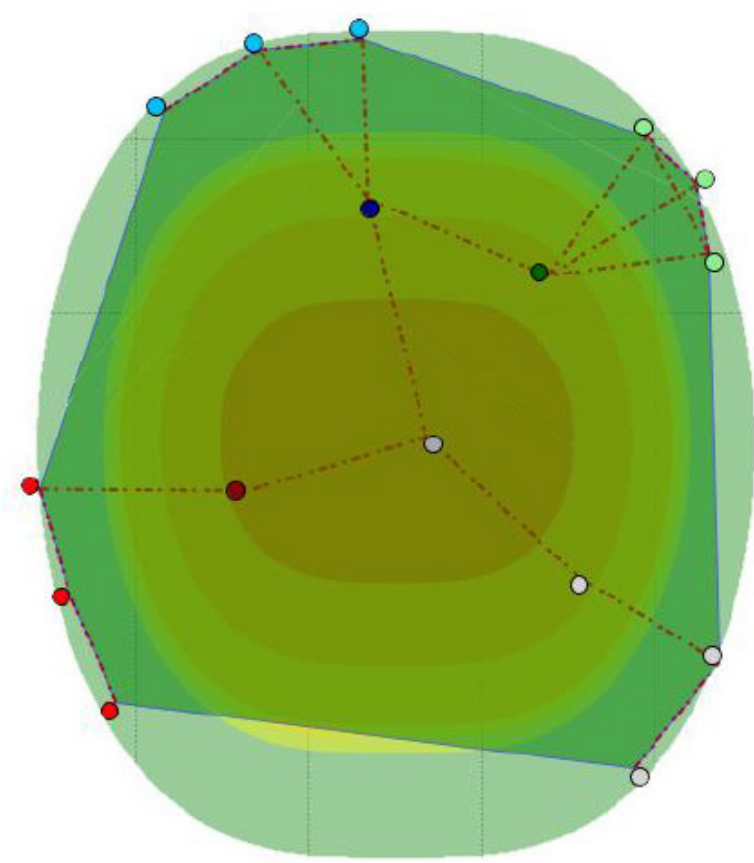

(b)

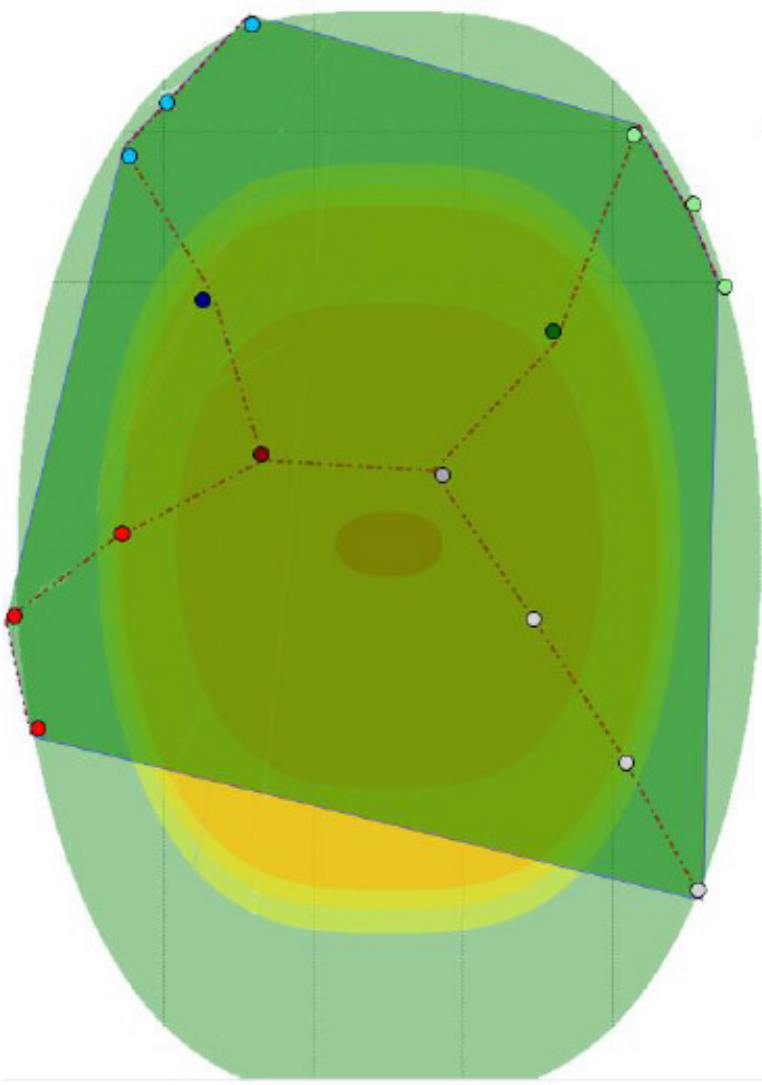

(d)

Fig. 6: Topology of the sensing network:(a) $t=52$, (b) $t=100$, (c) $t=180$ and (d) $t=220$. Nodes of the same cluster are marked with the same color, the cluster head is marked with darker hue. 
the reasonable values for the strategies parameters $r_{1}$ and $r_{2}$ are: $\frac{K}{2} \leq r_{1}<K$ and $\frac{K}{2}<r_{2}<K$. However, the future work should involve evaluation of the method performance based on simulation experiments for more complex scenarios with various number of clusters $K$.

\section{CONCLUSION}

MANETs can significantly enhance the capability to investigate contaminated areas, in particular detect and track phenomena clouds. In this paper we described the methodology for evaluating a quality of a network topology due to the possibility of determining a boundary of a cloud. The results of simulation experiments confirm that our approach can sufficiently support the process of detecting the temporarily optimal sensing devices configuration for cloud boundary monitoring at a given time. Unfortunately, our experimental results demonstrate that due to the dynamic nature of monitored phenomena clouds the quality of selected topology depends on the size of the observation time window and requirement of meeting all conditions for defined measurements. The trade-off between a quality of sensing network, time of calculation and fulfilling all requirements and expectations has to be assumed.

\section{ACKNOWLEDGMENT}

This article is based upon work from COST Action IC1406 High-Performance Modelling and Simulation for Big Data Applications (cHiPSet).

\section{REFERENCES}

[1] M. T. Thai, R. Tiwari, R. Bose, and A. Helal, "On detection and tracking of variant phenomena clouds," ACM Trans. Sen. Netw., vol. 10, no. 2 , pp. 34:1-34:33, Jan. 2014. doi: 10.1145/2530525

[2] T. Facchinetti, G. Franchino, and G. Buttazzo, "A distributed coordination protocol for the connectivity maintenance in a network of mobile units," in Sensor Technologies and Applications, 2008. SENSORCOMM'08. Second International Conference on. IEEE, 2008. doi: 10.1109/SENSORCOMM.2008.31 pp. 764-769.

[3] Z. Kan, L. Navaravong, J. M. Shea, E. L. Pasiliao, and W. E. Dixon, "Graph matching-based formation reconfiguration of networked agents with connectivity maintenance," Control of Network Systems, IEEE Transactions on, vol. 2, no. 1, pp. 24-35, 2015. doi: 10.1109/TCNS.2014.2367363
[4] N. Michael, M. M. Zavlanos, V. Kumar, and G. J. Pappas, "Maintaining connectivity in mobile robot networks," in Experimental Robotics. Springer, 2009. doi: 10.1007/978-3-642-00196-3-14 pp. 117-126.

[5] A. Konak, G. E. Buchert, and J. Juro, "A flocking-based approach to maintain connectivity in mobile wireless ad hoc networks," $A p$ plied Soft Computing, vol. 13, no. 2, pp. 1284-1291, 2013. doi: 10.1016/j.asoc.2012.10.020

[6] M. Krzyszton and E. Niewiadomska-Szynkiewicz, "Heavy gas cloud boundary estimation and tracking using mobile sensors," Journal of Telecommunications and Information Technology, no. 3, p. 38, 2016.

[7] E. Niewiadomska-Szynkiewicz, A. Sikora, and M. Marks, "A movementassisted deployment of collaborating autonomous sensors for indoor and outdoor environment monitoring," Sensors, vol. 16, no. 9, p. 1497, 2016. doi: $10.3390 / \mathrm{s} 16091497$

[8] M. Patan, Optimal sensor networks scheduling in identification of distributed parameter systems. Springer Science \& Business Media, 2012, vol. 425.

[9] M. Krzysztoń, "Comparison of manet self-organization methods for boundary detection/tracking of heavy gas cloud," in Computer Science and Information Systems (FedCSIS), 2016 Federated Conference on. IEEE, 2016. doi: 10.15439/2016F240 pp. 1075-1084.

[10] S. Srinivasan, S. Dattagupta, P. Kulkarni, and K. Ramamritham, "A survey of sensory data boundary estimation, covering and tracking techniques using collaborating sensors," Pervasive and Mobile Computing, vol. 8, no. 3, pp. 358-375, 2012. doi: 10.1016/j.pmcj.2012.03.003

[11] K. Shanmugam, K. Subburathinam, and A. Velayuthampalayam Palanisamy, "A dynamic probabilistic based broadcasting scheme for manets," The Scientific World Journal, vol. 2016, 2016. doi: $10.1155 / 2016 / 1832026$

[12] M. Król, E. Schiller, F. Rousseau, and A. Duda, "Weave: Efficient geographical routing in large-scale networks.” in EWSN, 2016, pp. 89100 .

[13] R. Virrankoski and A. Savvidees, "Tasc: topology adaptive spatial clustering for sensor networks," in Mobile Adhoc and Sensor Systems Conference, 2005. IEEE International Conference on. IEEE, 2005. doi: 10.1109/MAHSS.2005.1542850 pp. 10-pp.

[14] M. Zhao and W. Wang, "Analyzing topology dynamics in ad hoc networks using a smooth mobility model," in Wireless Communication and Networking Conference, 2007. WCNC 2007. IEEE. IEEE, 2007. doi: 10.1109/WCNC.2007.604 pp. 3279-3284.

[15] A. Sikora, E. Niewiadomska-Szynkiewicz, and M. Krzysztoń, "Simulation of mobile wireless ad hoc networks for emergency situation awareness," in Computer Science and Information Systems (FedCSIS), 2015 Federated Conference on. IEEE, 2015. doi: 10.15439/2015F52 pp. 1087-1095

[16] D. Morgan Jr, L. K. Morris, and D. L. Ermak, "Slab: a time-dependent computer model for the dispersion of heavy gases released in the atmosphere," Lawrence Livermore National Lab., CA (USA), Tech. Rep., 1983.

[17] E. Niewiadomska-Szynkiewicz, A. Sikora, and J. Kołodziej, "Modeling mobility in cooperative ad hoc networks," Mobile Networks and Applications, vol. 18, no. 5, pp. 610-621, 2013. doi: 10.1007/s11036013-0450-2 